\title{
Measuring verbal recall. II: The ITR score expressed as a ratio
}

JOSEPH F. FAGAN III, DEPARTMENT OF PSYCHOLOGY, CASE WESTERN RESERVE UNIVERSITY, Cleveland, Ohio 44106

The sequential consistency from one free recall sequence to another may be expressed as the ratio of observed bigram repetitions to the maximum possible minus the ratio of the expected number of bigram repetitions to the maximum possible.

Many investigators assume that the ability to organize incoming stimulation is an important factor in verbal learning. One estimate of organization is the degree to which $\mathrm{S}$ is consistent in ordering his responses over a series of free recalls when different permutations of the same items are presented from trial to trial. The fact that such response constancies develop was reported by Tulving (1962), who labeled the phenomenon "subjective organization," (SO). In the present paper, simply for the purposes of exposition, this consistency in response ordering in the free recall situation will be referred to as "sequential consistency" (SC).

There are two methods currently available for quantifying SC, the original SO score proposed by Tulving which was derived from information theory and the "intertrial repetition" (ITR) system of Bousfield \& Bousfield (1966). In both schemes, the basic unit of consistency is the recurring bigram. The replies HAT, DOG, HORSE, SHOE and HORSE, SHOE, HAT, DOG, for example, contain the repeated bigrams HORSE-SHOE and HAT-DOG. The two formulations differ, however, in their expression of SC. The SO system represents $\mathrm{SC}$ as the ratio of the obtained repetition to the maximum possible, while the ITR score more simply expresses $\mathrm{SC}$ as the number of repeated bigrams on two successive recalls.

For various practical and theoretical reasons, there are occasions when it is desirable to employ a ratio concept of SC. Unfortunately, there are two disadvantages to the SO ratio score; it is laborious to calculate, and there is no explicit way to determine what SO value to expect by chance. The purpose of the present report is to suggest that Bousfield \& Bousfield's ITR system may be adapted to represent $\mathrm{SC}$ in the form of a ratio. The rationale is as follows:

For a series of free recall trials where different permutations of the same items are presented from trial to trial and any two orderings of recall sequences are chosen at random, sequential consistency, SC, is equal to observed sequential consistency, $\mathrm{O}(\mathrm{SC})$, minus the amount of sequential consistency expected by chance, $\mathrm{E}(\mathrm{SC})$ :

$$
\mathrm{SC}=\mathrm{O}(\mathrm{SC})-\mathrm{E}(\mathrm{SC})
$$

Defining $\mathrm{O}(\mathrm{SC})$ as the ratio of the observed number of intertrial repetitions, O(ITR), to the maximum possible number of intertrial repetitions, MAX(ITR), and $\mathrm{E}(\mathrm{SC})$ as the ratio of the expected number of intertrial repetitions, E(ITR), to the maximum possible, Formula 1 becomes:

$$
\mathrm{SC}=\frac{\mathrm{O}(\mathrm{ITR})}{\mathrm{MAX}(\mathrm{ITR})}-\frac{\mathrm{E}(\mathrm{ITR})}{\mathrm{MAX}(\mathrm{ITR})}
$$

Thus, to determine SC, three values are necessary: O(ITR), $\mathrm{E}(\mathrm{ITR})$, and MAX(ITR). O(ITR) is simply the number of bigrams common to the two recalls. E(ITR) has been shown by Bousfield and Bousfield to be a function of three variables: $h$, the number of items recalled on trial $t ; k$, the number of items recalled on trial $t$ +1 ; and $c$, the number of items common to the two recalls. $E(I T R)=\frac{[c(c-1)]}{h k}$ (p. 938, Formula IV). MAX(ITR) between any two recalls, of course, is $\mathrm{c}-1$. Hence,

$$
\mathrm{SC}=\frac{\mathrm{O}(\mathrm{ITR})}{\mathrm{c}-1}-\frac{[\mathrm{c}(\mathrm{c}-1)]}{\frac{\mathrm{hk}}{\mathrm{c}-1}}
$$

and the expression

$$
\frac{[c(c-1)]}{\frac{h k}{c-1}} \quad \text { reduces to } \quad \frac{c}{h k}
$$

to yield:

$$
\mathrm{SC}=\frac{\mathrm{O}(\mathrm{ITR})}{\mathrm{c}-1}-\frac{\mathrm{c}}{\mathrm{hk}}
$$

To illustrate the $\mathrm{SC}$ score, consider the sequences $\mathrm{A}, \mathrm{D}, \mathrm{F}, \mathrm{B}, \mathrm{G}$, and $\mathrm{A}, \mathrm{D}, \mathrm{B}, \mathrm{F}$. In this example, $\mathrm{O}(\mathrm{ITR})=1$, since $\mathrm{A}-\mathrm{D}$ is the only repeated bigram, $c=4, h=5, k=4$, and $S C=1 / 3-1 / 5=.33$ $-.20=.13$. The present formulation should be particularly useful for measuring SC independently of sheer item recall, since the SC measure should be relatively invariant for different lengths of sequences.

\section{REFERENCES}

BOUSFIELD, A. K., \& BOUSFIELD, W. A. Measurement of clustering and of sequential constancies in repeated free recall. Psychol. Rep., 1966, 19, 935-942.

TULVING, E. Subjective organization in the free recall of "unrelated" words. Psychol. Rev., 1962, 69, 344-354.

NOTE

1. With the exception that $\mathrm{E}(\mathrm{SC})$ must be zero when $c$ equals 1 . 